\title{
Mental health treatment online during the COVID-19 outbreak
}

\author{
Nannan $\mathrm{Hu}^{1} \cdot$ Shengming Pan ${ }^{1} \cdot$ Jijun Sun ${ }^{1} \cdot$ Zhuo Wang $^{1} \cdot$ Hongjing Mao ${ }^{1}$
}

Received: 4 March 2020 / Accepted: 14 April 2020 / Published online: 2 May 2020

(c) Springer-Verlag GmbH Germany, part of Springer Nature 2020

Keyword COVID-19 · Mental health · Online treatment

The Coronavirus Disease-19 (COVID-19) broke out in the city of Wuhan, China, and rapidly spread out to other provinces and regions. Not only were neighbour countries infected, but it is now classed by WHO, a world-wide pandemic. Definite evidence has shown that the COVID-19 is mainly transmitted by person to person contact [1]. So far, more than 80,928 citizens have been infected, while 3245 have died in China by March 18, 2020. It was found that COVID-19 is $96 \%$ identical at the whole-genome level to a bat coronavirus [2]. The Chinese government has taken measures such as delaying return to work and postponing school start dates to help prevent cluster cases which might lead to the disease diffusing quickly throughout the population. Chinese government has also ordered the isolation of large regions and cities. Currently, the fast spread of the infection coupled with its high lethality has caused a level of panic in the general public.

Chloroquine phosphate, an old drug for treatment of malaria, has shown apparent efficacy with an acceptable level of safety against COVID-19 [3]. Also, traditional Chinese medicine proves to be effective [4]. In addition, other treatments are currently under investigation (e.g. the Ebola treatment remdesivir).

The outbreak of COVID-19 and its creation of public panic, coupled with the increasing number of patients and suspected cases, have caused real concern about the possibility of infection. Bao and his colleague emphasized that the mental health of the citizenry and the involved medical health-care workers urgently need this problem to be addressed [5]. The convenience of internet and smart phones

Communicated by Andrea Schmitt.

Hongjing Mao

13777861972@163.com

1 Hangzhou Seventh People's Hospital, Zhejiang University School of Medcine Mental Health Center, Hangzhou, China made it possible for psychiatrists and mental health centers to provide online mental health services during the COVID19 outbreak [6], to decrease patient cross-infection in hospitals, due to the online consultation provided.

Patients get access to Ali-pay, which is a mobile application developed by Jack Ma's team and widely used in China. Then they may search Zhejiang internet hospital platform (in Chinese), and choose an online consultation or internet phone consultation. Patients are able to upload their medical records, which can only be seen by the consulting doctor. It is a great comfort to patients with chronic diseases to access professional advice and get free assessment. There are also online lectures and articles about health education to help prevent any relapse in chronic mental patients. These resources guide citizens on how to relax at home, for example, they may use the knowledge gained from respiratory training and meditation. Aerobic exercise programs, for example, jogging, swimming, badminton, are recommended as they can help relieve adverse emotions. If conditions are restricted, leg raises, squats, push-ups, and abdominal exercises are also helpful.

Increasingly, psychologists and psychiatrists are utilizing the internet and social media such as WeChat and Weibo to share strategies for dealing with psychological stress [5]. Strategies such as (1) guide the public to recognize the epidemic correctly. Do not exaggerate the consequences. (2) Psychological reactions such as anxiety, depression, insomnia, and obsession may occur during the outbreak and how to respond to these reactions. (3) Keep up social connections. Chatting and communicating with family, friends, and co-workers through the use of phone or internet. To some individuals, pets can soothe emotions. Some patients may be satisfied with online consultation, while others may not. To those who really need psychopharmacological treatment for their mental illness, we suggest such patients who are living near the Hangzhou Seventh People's Hospital to come to the hospital at their earliest convenience. 
Despite the potent infectiousness, the tendency of COVID-19 is possible to be inhibited. Newly ascertained cases are decreasing in numbers daily, which means the Chinese government measures have been extremely effective. However, mental health care is urgently needed [7], especially for medical workers who faced extreme risk of becoming infected and high-pressure work caused by rescuing severely ill patients [8]. As for the current situation, networking of medical treatments is playing an important role in the development of medicines. Thus, online mental services should be further developed to help maintain support of mentally ill patients in major public health events like COVID-19.

\section{Compliance with ethical standards}

Conflict interests The authors declare no competing interests.

Ethics approval Not applicable.

\section{References}

1. Huang C, Wang Y, Li X et al (2020) Clinical features of patients infected with 2019 novel coronavirus in Wuhan. China
Lancet 395(10223):497-506. https://doi.org/10.1016/S0140 -6736(20)30183-5

2. Zhou P, Yang XL, Wang XG et al (2020) A pneumonia outbreak associated with a new coronavirus of probable bat origin. Nature. https://doi.org/10.1038/s41586-020-2012-7

3. Gao J, Tian Z, Yang X (2020) Breakthrough: Chloroquine phosphate has shown apparent efficacy in treatment of COVID-19 associated pneumonia in clinical studies. Biosci Trends. https:// doi.org/10.5582/bst.2020.01047

4. Luo H, Tang QL, Shang YX et al (2020) Can Chinese medicine be used for prevention of corona virus disease 2019 (COVID19)? A review of historical classics, research evidence and current prevention programs. Chin J Integr Med. https://doi.org/10.1007/ s11655-020-3192-6

5. Bao Y, Sun Y, Meng S et al (2020) 2019-nCoV epidemic: address mental health care to empower society. Lancet. https://doi. org/10.1016/S0140-6736(20)30309-3

6. Liu S, Yang L, Zhang C et al (2020) Online mental health services in China during the COVID-19 outbreak. Lancet Psychiatry. https ://doi.org/10.1016/S2215-0366(20)30077-8

7. Xiang Y, Yang Y, Li W et al (2020) Timely mental health care for the 2019 novel coronavirus outbreak is urgently needed. Lancet Psychiatry. https://doi.org/10.1016/S2215-0366(20)30046-8

8. Kang L, Li Y, Hu S et al (2020) The mental health of medical workers in Wuhan, China dealing with the 2019 novel coronavirus. Lancet Psychiatry. https://doi.org/10.1016/S2215 $-0366(20) 30047-X$ 